\title{
Staatliche Lohnarbeiter im Kapitalismus
}

\section{Gerhard Armanski}

Der aktuellen Diskussion über die Mittelklassenproblematik und auch diesem Beitrag liegt kein losgelöster wissenschaftlicher Ideenstreit zugrunde. Ihr geht die politische Erkenntnis voraus, daß der Sturz der Bourgeoisherrschaft auch und gerade im entwickelten Kapitalismus nicht allein durch die Arbeiterklasse erfolgen kann. Die für das notwendige Bündnis in Frage kommende Klasse ist nicht mehr die der Kleinbourgeoisie bzw. der Bauern. Das Kleinbürgertum und die Bauernklasse schrumpfen zahlenmäßig in den modernen bürgerlichen Gesellschaften unablässig zusammen, als Klasse drücken sie sich kaum noch geschlossen aus (1). In den Vordergrund der Bündnisfrage tritt heute die lohnarbeitende Mittelklasse, insbesondere die staatlichen Lohnarbeiter. In der Tatsache, daß es sich bei ihr ebenfalls um Lohnarbeiter handelt, spiegelt sich die Vernichtung des Kleinbesitzes und die mit der wachsenden kapitalistischen Vergesellschaftung der Produktion und Reproduktion fortschreitende Polarisierung der bürgerlichen Gesellschaft in Kapitalisten und Lohnarbeiter. So stellt sich die Bündnisfrage heute auf einer weiteren Stufe der gesellschaftlichen Entwicklung neu, nämlich nicht als Bündnis zwischen Arbeiterklasse und kleinen Warenproduzenten, Händlern usw., sondern als besonderes Bündnis zwischen zwei Klassen, deren Gemeinsamkeit die Lohnform ihrer Arbeitsverausgabung und die darin eingeschlossene Abpressung von Mehrarbeit ist. Gleichwohl gibt es erhebliche Unterschiede in der Stellung innerhalb der gesellschaftlichen Organisation der Arbeit und der ökonomischen Grundlage ihrer Existenz zwischen beiden Klassen, die zu unterschiedlichen Interessen und Bewußtseinsformen führen, und zwar in stärkerem Maß als dies zwischen den Fraktionen der Arbeiterklasse selbst der Fall ist.

Es geht nun darum, die Gemeinsamkeiten und die Unterschiede zwischen staatlichen Lohnarbeitern und Arbeiterklasse $\mathrm{zu}$ analysieren, um damit der Frage nach den Grundlagen und der Taktik eines Bündnisses zwischen beiden näherzukommen. Dabei wird sich zeigen, daß die wissenschaftliche Analyse und die politische Taktik in den vorhandenen Positionen zu dieser Frage eng zusammenhängen, was an einigen Beispielen aufgezeigt werden soll.

Allerdings ist die Kritik an diesen Positionen und die kurze Darstellung der eigenen Auffassung noch gebunden an die Ebene der Entfaltung des, allgemeinen Klassenbegriffs ${ }^{66}$. Zwar enthält dieser, wie die gesamte Kritik der politischen Ökonomie, die historische Realität der bürgerlichen Gesellschaft in ihrem Durchschnitt. Die Besonderheit einer bestimmten historischen Epoche, etwa der Geschichte der Bundesrepublik, schlïsselt er aber noch keineswegs zufriedenstellend auf. Insofern

1 Vgl. Annette Leppert-Fögen, Die deklassierte Klasse - Studien zur Geschichte und Ideologie des Kleinbürgertums, Frankfurt a. M. 1974; Zahlen in den Sozialstatistikbänden des Projekts Klassenanalyse und des IMSF. 
sind, damit Kritik und eigene Position vollständig entwickelt werden können, noch weitere Arbeitsschritte zu leisten. Einmal ist im Rahmen des ,,allgemeinen Klassenbegriffs" selbst noch das Verhältnis von Kapitalproduktion und Staat als Grundlage von Lage und Bewußtsein der staatlichen Lohnarbeiter über die bisherigen Ansätze hinaus zu klären. Weiterhin müssen sich natürlich die erarbeiteten Auffassungen in der wissenschaftlichen Analyse und politischen Einschätzung der gegenwärtigen Verhältnisse in der BRD (3) erst noch als richtig erweisen. Insofern stellt die nachstehende Arbeit nur einen ersten Schritt dar.

I.

In den letzten Jahren ist verschiedentlich auch von marxistischer Seite her der Versuch unternommen worden, die staatlichen Lohnarbeiter und die dem Kapital unterworfenen Lohnarbeiter als Abteilungen einer gemeinsamen Klasse - der Lohnarbeiterklasse - zu fassen (4). Für diese Position stellte sich anstelle der Bündnisfrage die Frage der Vereinheitlichung aller Lohnarbeiter - und zwar ausgehend von der Gemeinsamkeit der Lohnform ihrer Arbeit.

Die Lohnform wird zum klassenbestimmenden Merkmal und alle Lohnarbeiter in der Produktion, im Handel und beim Staat als einer Klasse zugehörig erklärt. Die Argumentation beruft sich auf Marx, der im letzten Kapitel des „Kapital“ davon spricht, daß

„die Eigentümer von bloßer Arbeitskraft, die Eigentümer von Kapital und die Grundeigentümer, deren respektive Einkommensquellen Arbeitslohn, Profit und Grundrente sind, also Lohnarbeiter, Kapitalisten und Grundeigentümer ... die drei großen Klassen der modernen, auf der kapitalistischen Produktionsweise beruhenden Gesellschaft (bilden)" (5).

Aus dem Zusammenhang der genannten Stelle geht aber eindeutig hervor, daß MarX hier nur die vom Kapital angewendeten Lohnarbeiter meinen kann. Mit dem entsprechenden Kapitel schließt nämlich die Untersuchung des kapitalistischen Reproduktionsprozesses in seiner Gesamtheit ab, innerhalb dessen die aus abgeleitetem Einkommen (Revenue) gespeiste ökonomische Existenz des Staates und damit der staatlichen Lohnarbeiter noch gar nicht entwickelt ist.

Die Theorie der Lohnarbeiterklasse sitzt genau der Verschleierung wesentlicher Verhältnisse an der Oberfläche der bürgerlichen Gesellschaft auf, die Marx

2 Vgl. als Versuch hierzu: Gerhard Armaski, Boris Penth und Jörg Pohlmann, Situation und Kämpfe staatlicher Lohnarbeiter in der BRD, erscheint voraussichtlich Januar 1975.

3 Vgl. Gerhard Armanski, Zur Kritik der Theorie der neuen Mittelklasse, in: Probleme des Klassenkampfs, 4/1972; Autorenkollektiv, Zur Klassenanalyse der Studenten, Erlangen 1972. Die hier vertretene Position wurde vom Verf. des vorliegenden Aufsatzes selbst geteilt. Sie hatte in der Abgrenzung von Analysen, die das Begriffspaar produktive und unproduktive Arbeit unbesehen als Instrumente der realen Klassenanalyse verwendeten (vgl. die Arbeiten Schmierers), einen bestimmten wissenschaftlichen und politischen Stellenwert, ist aber heute in entscheidenden Punkten als unrich tig anzusehen.

4 MEW 25, S. 892 (MEW - Marx/Engels Werke)

$5 \quad$ Vgl. MEW 23, S. 555 
analysierte. Bereits im Lohn ist der die kapitalistische Produktion kennzeichnende Unterschied von notwendiger und Mehrarbeit ausgelöscht. Der Lohn ist die Form, die den Inhalt - die Ausbeutung der Ware Arbeitskraft - vermittelt und versteckt (6). Diese Form existiert auch im Waren- und Geldhandel, wo es allerdings ein gesellschaftlich anders bestimmtes Ausbeutungsverhältnis verhüllt (7). Mit der weiteren Verselbständigung von Teilen der gesellschaftlich produzierten Mehrwertmasse im zinstragenden Kapital (Trennung von ,arbeitendem“, fungierendem und Leihkapital) setzt sich z. B. im Unternehmerlohn (8) die Lohnform auch für Verhältnisse fest, die ihrem Inhalt, der Ausbeutung in Produktion und Zirkulation direkt widersprechen. Schließlich erscheint den das Kapitalverhältnis in Produktion und Zirkulation reproduzierenden Lohnarbeitern der Lohn nicht nur als Preis ihrer Arbeit, sondern als spezifische Revenue (Einkommen), die ihnen als Eigentümer ihrer Arbeitskraft neben den aus dem Boden und dem Kapital stammenden Revenuen zukommt (9). In der Revenueform ist die gesellschaftliche Bestimmung der Lohnarbeit als wert- und mehrwertproduzierende bzw. Zirkulationsarbeit verrichtende Lohnarbeit ausgelöscht. Der Lohn erscheint bloß noch als Vergütung für ein entsprechendes Arbeitsquantum.

Bisher war nur vom Lohn als Einkommen der in der Reproduktion des Kapitals beschäftigten Lohnarbeiter die Rede. Ihre Revenuen (Einkommen) sind Teile des produzierten Kapitals - Originalrevenuen. Anders bei den staatlichen Lohnarbeitern. Ihr Lohn setzt sich aus Teilen der Originalrevenuen der Bourgeois und der Arbeiter (Profit und Lohn) zusammen. Sie produzieren ihren Lohn nicht selbst (was allerdings auch die kommerziellen Lohnarbeiter nicht direkt tun), reproduzieren damit auch nicht das Kapitalverhältnis. Sie stehen nicht dem Kapitalisten gegenüber, der am Gebrauchswert ihrer Arbeit für die Wertproduktion interessiert ist. Sie stehen dem Staat gegenüber, der am Gebrauchswert ihrer Arbeit zur Erfüllung seiner Funktionen interessiert ist.

Der Lohn stellt das Äquivalent für die Reproduktionskosten der Ware Arbeitskraft dar. Im Ankauf des Lohnarbeiters ist das Recht und der Zweck eingeschlossen, die gekaufte Ware Arbeitskraft möglichst profitabel anzuwenden. Die Mehrarbeitsabpressung als Inhalt des Lohnarbeitsverhältnisses tritt in ihrer reinsten Form als Mehrwertproduktion durch den produktiven Lohnarbeiter in der Industrie auf, verallgemeinert sich jedoch mit der Durchsetzung der kapitalistischen Produktionsverhältnisse bis hin in Sphären, in denen nicht kapitalistisch produziert wird, also etwa beim Staat. Mit der Ferne zum Reproduktionsprozeß des Kapitals als sich selbst verwertender Wert verschwindet aber die der Lohnarbeit anhaftende Bestimmung, das Kapital zu reproduzieren und zu vermehren. Der Lohn ist auch in der

Vgl. MEW 25, S. $278 \mathrm{ff}$.

Vgl. MEW 25, S. $393 \mathrm{ff}$.

Vgl. MEW 25, S. 822 ff.

Das Projekt Klassenanalyse verschüttet das Problem lediglich, wenn es behauptet, sobald die Arbeitskraft der staatlichen Lohnarbeit als tauschwertbestimmt und mehrarbeitsleistend angesehen werde, sei die Differenz zum Ankauf und Gebrauch der Lohnarbeit in der Kapitalproduktion nicht mehr recht einzusehen. Vgl. Materialien zur Klassenstruktur der BRD, S. 493 f. Leider bleibt es auch bei der bloßen Behauptung. 
Sphäre des Staates das Aquivalent für die Reproduktionskosten der Ware Arbeitskraft. Deren Vernutzung im Arbeitsprozeß geschieht mit dem Zweck der Herstellung für die bürgerliche Gesellschaft nützlicher Dienste und nicht der Vermehrung des vorgeschossenen Kapitals. Daß diese Arbeitsverausgabung über die notwendige Arbeit hinaus Mehrarbeit leistet, liegt in der benannten, von der Kapitalproduktion ausgehenden und bestimmten Tendenz der Verallgemeinerung der Lohnarbeit, ist aber nicht Sinn und Zweck der ganzen Veranstaltung (10).

Die Zusammenfassung der verschiedenen Kategorien von Lohnarbeitern in einer Klasse bindet die Klassenbestimmung an die Oberfläche des gewöhnlichen Bewußtseins, in der die Quelle der verschiedenen Einkommen (der Arbeit, des Kapitals, des Grundbesitzes) nicht mehr sichtbar ist. Was sich selbst als eine die innere Differenzierung der Lohnarbeiter auslöschende Einheit verschiedener ökonomischer Bestimmungen von Lohnarbeit darstellt, kann nicht Ausgangspunkt der Klassenanalyse sein. Die Theorie der Lohnarbeiterklasse ist daher als klassenanalystischer Begriff kaum, als politischer Kampfbegriff (Vereinheitlichung der Lohnarbeiterklasse) überhaupt nicht brauchbar. Auf die mannigfachen Kämpfe der Lohnarbeiter weiß sie nur mit einer Vereinheitlichungsstrategie auf der Basis des Lohns zu antworten, verliert dabei aber die Frage nach der objektiven revolutionären Kraft, die vom Kapital erzeugt wird und die allein es vernichten kann, aus dem Auge. Unterschiede und Gemeinsamkeiten zwischen Arbeiterklasse und staatlichen Lohnarbeitern können dann nur noch zufällig auf der Ebene der Erscheinungen festgemacht, nicht aber systematisch erklärt und hergeleitet werden (11).

Als Ausgangspunkt der Klassenanalyse ist vielmehr die spezifisch kapitalistische Form der Produktion und Verteilung des gesellschaftlichen Reichtums anzusehen, die verschiedene Formen der Lohnarbeit hervorbringt. In der kapitalistischen Produktionsweise stehen sich die Klasse der Arbeiter, die nur leben können, wenn sie das Kapital vermehren und die Klasse der Kapitalisten, die die aneignende Nicht-Arbeit, das Kapital, repräsentieren, gegenuiber. Die vom Kapital ausgebeuteten Lohnarbeiter, die mit ihrer Arbeit in Produktion und Handel das Kapitalverhältnis reproduzieren, stellen objektiv die Arbeiterklasse dar. Sie, als Gegenpol des Kapitals, sind auch Träger der historischen Aufgabe der Aufhebung des Kapitalverhältnisses; die aus abgeleiteter Revenue bezahlten und und nicht (wie die kommerziellen Lohnarbeiter) in den Kapitalreproduktionsprozeß eingeschlossenen staatlichen Lohnarbeiter können dann keine Abteilung der Arbeiterklasse sein.

II.

Nicos Poulantzas (12) unterscheidet eine ökonomische, eine strukturelle und eine

10 Vielleicht ist eine materialistische Basis für das Aufleben einer derartigen Theorie darin zu sehen, daß mit ihrer Hilfe die sozialistischen Studenten bzw. Intellektuellen sich selbst zur revolutionären Klasse zählen zu können hofften.

11 Zum marxistischen Klassenbegriff, Berlin (West) 1973. Vgl. auch Political Power and Social Classes, London 1973

12 Zum marxistischen Klassenbegriff, S. 12 
konjunkturelle Klassenbestimmung, die zusammen als klassenanaly tisches Kriterium anzuwenden sind. Bezüglich der ökonomischen Klassenbestimmung geht er davon aus, daß die produktive Lohnarbeit die Arbeiterklasse definiere. Der Lohn sei nur juristische Form des Einkommens, die unterschiedliche ökonomische Verhältnisse verdecke. ,Nicht der Lohn definiert die Arbeiterklasse“ (13), Poulantzas kritisiert die Theorie der KPF vom Gesamtarbeiter als ,ökonomistische Deformation“ (14). Unter den Gesamtarbeiterbegriff fielen außer den produktiven Arbeitern auch alle übrigen Lohnarbeiter, die „Quasi-Arbeiter“. Die Identifizierung von Lohnabhängigkeit und Arbeiterklasse führe zum „Mythos der Klasse der Lohnempfänger“.

Freilich gebe es neben den Hauptklassen weitere Klassen, wie das traditionelle Kleinbürgertum und das ,, ,neue Kleinbürgertum der nicht-produktiven Lohnempfänger" (15). Die Theorie von einer einheitlichen Zwischenklasse aus beiden als dritte Kraft lehnt Poulantzas jedoch ab. Vielmehr handle es sich hier um mehrere Klassen. Ebenso lehnt er ihre Fassung als Zwischenschichten ab. Bei der Hinzuziehung politisch-ideologischer Kriterien sei gleichwohl davon auszugehen, daß das traditionelle Kleinbürgertum und das neue, aus nichtproduktiven Lohnempfängern, „Funktionären des Staates und seiner verschiedenen Apparate" (16) bestehende Kleirıbürgertum eine Klasse bildeten.

„Das ,Kleineigentum einerseits und Lohnempfänger, die ihrer Ausbeutung nur in Form ,des ,Lohnes" und ,der Konkurrenz “ " abseits von der Produktion ausgesetzt sind, andererseits weisen aus gleichwohl unterschiedlichen ökonomischen Gründen die gleichen politischen und ideologischen Merkmale auf: kleinbürgerlicher ,Individualismus', Neigung zum ,Status quo' und Furcht vor der Revolution, Mythos des, gesellschaftlichen Aufstieges" und Streben nach bürgerlichem Status, Glaube an den ,neutralen Staat" über den Klassen, politische Unbeständigkeit und Tendenzen, den ,starken Staat', sowie Bonapartismus, Formen der Revolte vom Typ ,Aufstand der Kleinbürger" zu unterstützen"

Diese relativ einheitliche Kleinbürgerklasse zerfalle freilich in ökonomisch bestimmte Fraktionen und eher politisch-ideologisch bestimmte Schichten. Es lasse sich feststellen, ,daß die kleinbürgerliche Fraktion der nicht-produktiven Lohnempfänger der Arbeiterklasse näher ist als die des traditionellen Kleinbürgertums“. Im übrigen schwankte die Nähe der jeweiligen Fraktion zur Arbeiterklasse nach der Konjunktur. Auf keinen Fall aber seien die nicht-produktiven Lohnempfänger eine neue Klasse oder Zwischenschicht neben und außer den Klassen. Bei noch stärkerer Heranziehung politisch-ideologischer Kriterien sei von ,sozialen Kategorien“ auszugehen, z. B. der der administrativen Bürokratie, die trotz verschiedener Klassenbasis jeweils eine Interesseneinheit bilden und eine eigene Rolle als ,soziale Kräfte“ spielen könnten (19).

A. a. O., S. 14

A. a. O., S. 17

A. a. O., S. 24

A. a. O., S. 25

entf.

entf.

Vgl. a. a. O., S. 28 ff. Als ,soziale Kategorien (bezeichnet man) Gesamtheiten von 
Poulantzas versucht also, einer ökonomisch verengten Klassendefinition zu entgehen, indem er strukturelle und politisch-ideologische Kriterien in sie aufnimmt. In diesem Versuch der Verbindung wesentlicher und oberflächlicher Klassenkriterien landet Poulantzas schließlich auf der Ebene der Verallgemeinerung historischer Phänomene. Er beläßt es bei der bündigen Gleichsetzung von produktiver Arbeit und Arbeiterklasse. Damit fallen nicht nur die kommerziellen Lohnarbeiter heraus. Es werden auch Form und Inhalt der Lohnarbeit beim Staat überhaupt nicht entwickelt (20), wird nicht angegeben, welche Bedeutung die Lohnform für die Staatsarbeiter hat. Aus dem Widerspruch, die staatlichen Lohnarbeiter nicht einer einheitlichen Zwischenklasse zuzuschlagen, sie aber auch nicht als neue gesellschaftliche Klasse sehen zu wollen, flüchtet Poulantzas in die Konstruktion einer politisch-ideologisch bestimmten Kleinbürgerklasse. Die für sie angegebenen Kriterien mögen bis zu einem gewissen Grad zutreffende Verallgemeinerungen historisch entwickelter Bewußtseinsformen von traditioneller Kleinbourgeoisie und staatlichen Lohnarbeitern sein, eine aus der Anatomie der bürgerlichen Gesellschaft gewonnene Klassenanalyse ersetzen sie keinesfalls. Die Vermengung von Versatzstücken einer wissenschaftlichen Analyse mit allgemein gesetzten empirischen Beobachtungen macht klare politi-che Folgerungen unmöglich. So ist es denn auch kein Wunder, daß Poulantzas zur „Frage der Bündnisse der Arbeiterklasse mit den anderen Volksklassen“ (21) nur zu sagen weiß, daß ein „Bündnis ohne Prinzipien“(22) gefährlich ist.

III.

Heinz Jung (23) geht zunächst davon aus, daß sich die Kleinbourgeoisie, denen ursprünglich auch die Staatsbeschäftigten zugehörten, durch zunehmende Proletarisierung aufgelöst und differenziert habe (24). U. a. deswegen bilde die Kleinbourgeoisie heute keine Klasse mehr, sondern „Mittel- oder Zwischenschichten“. Teile von ihnen seien zur Arbeiterklasse zu rechnen, deren Kern die „Industriearbeiterschaft der Großindustrie ... und des Bergbaus“ sei, so u. a. die „Untergruppen der Staatsangestellten und -beamten" (25). Andererseits aber gehörten wiederum Intelligenz und "lohnabhängige Zwischenschichten" zu den „Schichten zwischen den Klas-

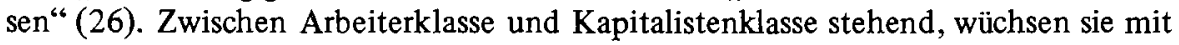

Agenten, deren hauptsächliche Rolle darin besteht, die Apparate des Staates und der Ideologie funktionieren zu lassen". S. 28

20 Wenn man nicht die Fassung des Staates als ,aus dem repressiven Apparat und den ideologischen Apparaten“ zusammengesetzt für ausreichend ansieht. A. a. O., S. 40

21 A. a. O., S. 17

22 A. a. O., S. 18

23 Zu den klassentheoretischen Grundlagen einer sozialstatistischen Analyse der Klassenund Sozialstruktur der BRD, in: IMSF (Hrsg.), Klassen- und Sozialstruktur der BRD 1950-170, Teil I: Klassenstruktur und Klassentheorie, Frankfurt a. M. 172, S. 1-192

A. a. O., S. 39 u. 42

A. a. O., S. 45 . Vgl. S. $128 \mathrm{ff}$.

A. a. O., S. 45 
dem Wachstum der gesellschaftlichen Vermittlungsbereiche (geistige Produktion und Staat) an. Arbeitsrechtlich und sozialökonomisch seien sie äußerst heterogen. So bildeten etwa untere Post- oder Eisenbahnbeamte eine „Randschicht der Arbeiterklasse“, während untere Polizeibeamte durch ihre Loyalitätsbindung und Herrschaftsfunktion eine „lohnabhängige Zwischengruppe" darstellten (27). Andererseits geht Jung davon aus, daß ,der zunehmende Umfang der Lohnarbeit nicht identisch (ist) mit der Zunahme der Arbeiterklasse“, daß der Ansatzpunkt zur ökonomischen Bestimmung der Arbeiterklasse vielmehr in der produktiven Arbeit liege (28).

Mit dem so entwickelten begrifflichen Instrumentarium geht Jung an die konkrete Analyse der BRD. Schlüsselbegriff für die Analyse der staatlichen Lohnarbeit sind die lohnabhängigen Mittelschichten, ,deren Existenzgrundlage die Lohnarbeit ist, deren Stellung in der gesellschaftlichen Organisation der der Arbeit und im Gesamtsystem der Wirtschaft und Gesellschaft ihnen jedoch gegenüber der Arbeiterklasse eine Sonderrolle zuweist. Hieraus resultiert eine dem ,alten' Kleinbürgertum vergleichbare objektive Stellung und eine darauf beruhende zwiespältige Interessenlage gegenüber den Grundklassen" (29). Zu ihnen gehören auch „Gruppen, die Funktionen des Staates ausüben“. Zur Lohnform ihrer Tätigkeit steht der inhaltliche Charakter (vor allem der repressive) ihrer Arbeit im Gegensatz. Die lohnabhängigen Mittelschichten seien durch rasches Wachstum und starke soziale Differenzierung gekennzeichnet. In ihren unteren Rängen bildeten sich „Detailarbeiter" heraus, die sich in Arbeitssituation, Qualifikation und Einkommen an die Arbeiterklasse annähern, wobei auch ihre zunehmende Rekrutierung aus dieser eine Rolle spiele. Bei diesen unteren Rängen ,setzt sich gegenüber der Zwiespältigkeit ihrer Funktionen der voll entfaltete Warencharakter ihrer Arbeitskraft durch" (30).

Der staatsmonopolistische Kapitalismus versuche, den Sonderstatus der lohnabhängigen Mittelschichten zu zementieren, müsse aber zugleich zunehmend ihre materiellen Privilegien abbauen. An sich Stützen des staatsmonopolistischen Kapitalismus, seien sie verschärftem Ausbeutungsdruck und zunehmender Existenzunsicherheit ausgesetzt. „Das führt bei beträchtlichen Gruppen zu einer, wenn auch widersprüchlichen und schwankenden Annäherung ihrer Interessen an die der Arbeiterklasse“ (31). Die lohnabhängigen Mittelschichten und die Intelligenz seien die neuen „, Nachbarn von rechts" “ (32) der Arbeiterklasse.

Die Staatsbeschäftigten werden nun nicht insgesamt unter der Kategorie der lohnabhängigen Mittelschichten gefaßt. Der Staat sei ,für eine große Gruppe der Arbeiterklasse, der lohnabhängigen Mittelschichten und der Intelligenz der unmittelbare Ausbeuter geworden" (33). Die Staatsbeschäftigten seien nach Arbeitsrecht und Funktion sehr unterschiedlich.

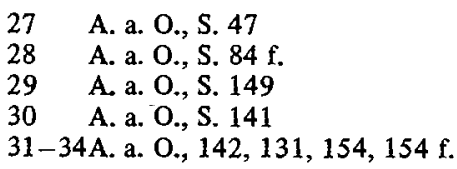


„Die Staatsbeschäftigten haben einen arbeitsrechtlich unterschiedlichen Beschäftigungsstatus..., sie üben qualitativ verschiedene Funktionen aus (Wirtschaftstätigkeit, Verwaltung, Repression usw.) und befinden sich schließlich in einer unterschiedlichen und gegensätzlichen Klassenlage (Arbeiterklasse, Mittelschichten, Intelligenz, Bourgeoisie, staatsmonopolistische Oligarchie)" (34).

Die hierarchische Struktur bringe soziale Gegensätzlichkeiten nur vermittelt zum Ausdruck. Beamtenstatus, materielle Privilegien, Loyalitätsverpflich tung seien noch immer für die Staatsbeschäftigten kennzeichnend. Ihre unteren Gruppen gehörten aber aufgrund ihrer Arbeits- und Lebensbedingungen zur Arbeiterklasse (35).

Auch Jung gibt die Analyse der Formen gesellschaftlicher Arbeit im Kapitalismus auf zugunsten empirisch gewonnener Verallgemeinerungen(36). Statt die historische Erscheinung konkreter Arbeits- und Lebensbedingungen der Lohnarbeiter mithilfe der Kritik der politischen Ökonomie zu durchdringen, zu erklären und $\mathrm{zu}$ ordnen, verfällt Jung in ein heilloses Durcheinander von stofflichen (konkreter Inhalt der Arbeit), historischen (Organisation, Kampfkraft, ökonomische Situation) und formmäßigen (Lohn, Rolle der Verausgabung der Arbeitskraft im Gesamtprozeß der Gesellschaft) Faktoren. Die Vermittlung von Wissenschaft und Erscheinung, Theorie und Geschichte in der Analyse der sozialen Klassen verflacht zur Sozialstrukturanalyse, die nurmehr begriffslos registriert statt zu erklären.

Jung kann nicht mehr analytisch klar angeben, wie die lohnabhängigen Mittelschichten bzw. die Staatsbeschäftigten im Verhältnis zur Arbeiterklasse zu begreifen sind. Mal sind sie eigene Schicht, mal soziale Gruppe, mal Teil der Arbeiterklasse je nach der historischen Erscheinung und dem von Jung verwendeten begrifflichen Instrumentarium. Wesentliche Bestimmungsfaktoren der staatlichen Lohnarbeit müssen so verborgen bleiben. Folgenschwer ist insbesondere, daß Jung an keiner Stelle Form und Funktion des bürgerlichen Staates ableitet (37). Damit kann die spezifische Quelle des Lohns der staatlichen Lohnarbeiter, nämlich abgeleitete Revenue, nicht mehr erkannt werden. Die besondere Herkunft des Lohns der Staatsarbeiter ist aber eine zentrale Bestimmung, aus der ihr Verhältnis zum Reproduktionsprozeß und den Lohnarbeitern des Kapitals, ihre spezifische Bindung an den Staat und damit die genauere Fassung der Besonderheiten der Lohnform ihrer Arbeit zu entwickeln sind. Die Besonderheit des Lohns der staatlichen Lohnarbeiter und die Bindung des Staats und seiner Funktionen an die Kapitalakkumulation erschließen erst den Zugang zur Analyse der staatlichen Lohnarbeiter. Diese stattdessen zu Schichten, sozialen Gruppen oder zur Arbeiterklasse zu schlagen, bedeutet eine Kapitulation vor dem Problem. Die Bündnisfrage löst sich dann letztlich dahin auf, $\mathrm{da} ß$ die staatlichen Lohnarbeiter in wesentlichen Teilen zur Arbeiterklasse gezählt

35 Vgl. a. a. O., S. 130

36 Zur Kritik an Jung vgl. Projekt Klassenanalyse, Materialien, S. 370-430. Die Behauptung der Genossen, aus der Kritik könne die Theorie des staatsmonopolistischen Kapitalismus ausgeklammert werden, geht allerdings an deren Bedeutung für die Jungsche Analyse vorbei, die aus vielen Stellen verbal und kausal ersichtlich wird. Zur Kritik an Jung vgl. Kommunist 4/5, KB/ML Westberlin 1971, S. $93 \mathrm{ff}$.

37 Das erklärt vielleicht, warum die mit Gesundheit, Erziehung und Sozialwesen befaßten staatlichen Lohnarbeiter in der Jungschen Analyse überhaupt nicht auftauchen. 
werden, während andere Teile diffus im sozialstrukturellen Raum verbleiben, bzw. in das antimonopolistische Bündnis eingereiht werden (38).

Das vorwiegend historische Erscheinungen verallgemeinernde Vorgehen von Jung muß an Fragen scheitern, die die reale Klassenkampfbewegung stellt, wie etwa des sehr unterschiedlichen und schwankenden Verhaltens der staatlichen Lohnarbeiter und ihrer Teile zur Arbeiterklasse. Einheit der Arbeiterklasse kann dann nur noch naiv und zufällig auf Angleichungstendenzen in der Arbeits- und Lebenslage gegründet werden, nicht aber auf die Bestimmungen und Tendenzen der Kapitalproduktion. Es ist auch weder wissenschaftlich notwendig noch politisch haltbar, ,, ungeklärte Reste ${ }^{\text {" }}$ in ein noch ungeklärteres Bündnis zu werfen, es sei denn, man hielte den Kampf gegen die Monopole für eine haltbare Grundlage proletarischer Bündnispolitik.

IV.

Der Ansatz des Projekts Klassenanalyse (39) unterscheidet sich wesentlich von Poulantzas bzw. Jung. Ausgehend von der Produktion und Verteilung des gesellschaftlichen Wertprodukts, gelangen sie dazu, die staatlichen Lohnarbeiter als Teil der aus Revenue (der Kapitalisten und der Lohnarbeiter) bezahlten ,dienstleistenden“ Lohnarbeiter zu begreifen, die sie deutlich von der Kleinbourgeoisie trennen. Es werden die Bedingungen des Wachstums der aus Revenue bezahlten Lohnarbeiter auseinandergelegt (40) und die charakteristischen Bewußtseinsmomente der dienstleistenden Lohnarbeit entwickelt (41). Allerdings kann wohl nicht die Rede davon sein, daß der Verwertungszwang des Kapitals für die unproduktiven Lohnarbeiter überhaupt keine Rolle spiele (42), daß diese in einem ,gemütlichen“ Arbeitsverhältnis stünden. Dem widerspricht auch, daß wenig später vom indirekten Niederschlag des industriellen Zyklus auf die Situation der unproduktiven Lohnarbeiter und der Bedeutung der Lohnform auch für die unproduktive Arbeit gesprochen wird (44).

Die staatlichen Lohnarbeiter werden in die Reihen der ,,dritten Personenru-

38 Hieran wird deutlich, daß3 die DKP-Klassenanalyse neben ihrer wissenschaftlichen Unzulänglichkeit immer wieder von politischen Setzungen überlagert wird, hier von einer historisch entwickelten Bündniskonzeption, welche die Volksfront im Gewand der antimonopolistischen Demokratie wiedererstehen lassen will mit einem spezifischen Klassenbündnis, in dem neue Zwischenschichten oder -klassen mitsamt der Kleinbourgeoisie und der nichtmonopolistischen Bourgeoisie die Bündnispartner des Proletariats bilden. Vgl. Christoph Möcklingshoff, Zur Bündniskonzeption von KPD und DKP, in: Probleme des Klassenkampfs, 4/1972

39 Materialien zur Klassenstruktur der BRD, Erster Teil - Theoretische Grundlagen und Kritiken, Berlin (West) 1973, S. 269 ff.

40 A. a. O., S. $271 \mathrm{ff}$.

41 A. a. O., S. $280 \mathrm{ff}$.

42 Wie auf $\mathrm{S} .283$ behauptet.

43 enti.

44-45A. a. O., S. 284 f., $288 ; 274$ 
briken oder Zwischen- und Mittelklassen" eingereiht, stellten weiterhin einen wesentlichen Teil der unproduktiven Lohnarbeiter dar. Die Untersuchung zeige,

„daß ihre Situation als Lohnarbeiter sie in Arbeits- und Lebensbedingungen stellt, die denen der produktiven Arbeiter in wesentlichen Zügen gleichen. Andererseits jedoch tauschen diese Arbeiter ihre Arbeitskraft nicht gegen Kapital, sondern reproduzieren sich aufgrund vom Profit etc. abgeleiteter Revenue. Sie fungieren also nicht im Produktions- und Reproduktionsprozeß des Kapitals; allein dieser - zweifellos gewichtige - Unterschied zu den produktiven Arbeitern macht es notwendig, sie mit ökonomisch ganz anders bestimmten Schichten unter dem Begriff der Mittelklassen zusammenzufassen“" (45).

Die starke Heterogenität dieser Mittelklassen lasse es nicht zu, sie als einheitliche dritte Klasse zu begreifen. Ihr Gemeinsames sei allein ihre „Existenz außerhalb des Kapitalverhältnisses“, was sowohl auf Kleinbürger wie aus abgeleiteter Revenue bezahlte Lohnarbeiter zutreffe.

Über diese allgemein richtigen Bestimmungen hinaus bringt die Analyse keine wesentlichen bzw. teils falsche Aufschlüsse iuber die staatlichen Lohnarbeiter. Mit den Hinweisen, daß die Arbeitsbedingungen beim Staat ,auf der politischen Ebene ein Abbild des kapitalistischen Fabriksystems" (46) seien, daß über den Staat die ökonomische Bewegung nur vermittelt auf die staatlichen Lohnarbeiter wirke (47), ist noch nicht viel gewonnen. Wichtiger ist schon die Erkenntnis, daß die staatlichen Lohnarbeiter, gerade weil sie von Profit- bzw. Lohnteilen (der Lohnarbeiter des Kapitals) leben, sowohl zu den Kapitalinteressen wie zu den Interessen der Arbeiterklasse im Gegensatz stehen.

„Der letzte Gegensatz wird sich in dem Maße im Bewußtsein der Lohnarbeiterklasse auflösen, wie sich die Einsicht in die Gleichartigkeit der Lebenslage durchsetzt und in der Forderung ausdrück t, nicht die Steuerlast der produktiven Arbeiter zu erhöhen, sondern die des Kapitals, wodurch der vermittelte ökonomische Gegensatz auch der unproduktiven Arbeiter zum Kapital rein hervortreten muß“ (48).

Unter dem Anspruch, eine begrifflich allgemeine Darstellung zu geben, schimmert allerdings die Gestalt der konkreten staatlichen Lohnarbeit in der BRD durch, bzw. nur eines Teils von ihr, nämlich der Beamten in der allgemeinen Verwaltung. Vom „Festgerittensein in eine bestimmte Arbeitsart“ (49) kann bei den staatlichen Lohnarbeitern weder historisch noch begrifflich die Rede sein, was sich gerade aus der Allgemeinheit und Abstraktheit staatlicher Tätigkeit ergibt. Hier wird die Bindung an den konkret-nützlichen Charakter der Arbeit, Arbeitsweise und Arbeitsinhalt also, mit der sozialen Standortbestimmung in der Organisation der Arbeit in eins gesetzt. Auch die Differenzierung der staatlichen Lohnarbeiter nach Funktion und Status ist unzureichend. Das schlägt sich z. B. in der Behauptung nieder, die Staatsagenten seien freie Lohnarbeiter, wobei die wesentliche Einschränkung der freien Beweglichkeit der Lohnarbeit etwa bei Beamten herausfällt. Derartige Ungenauig-
A. a. O., S. 294
A. a. O., S. 297
A. a. O., S. 296
A. a. O., S. 298 
keiten und Fehler werden mit der ansonsten sehr seltenen Forderung des „Übergangs in die empirische Untersuchung " $(50)$ nur zugedeckt.

Norbert Kostede (51) kritisiert an der Lohnarbeiterklassentheorie, daß sie „die spezifische Relevanz des Begriffs der Mittelklassen verkennt und diesen Begriff als im Rahmen einer marxistischen Klassentheorie überflüssig erscheinen läßt", ihn durch „den alles umfassenden ,Oberflächenbegriff von Lohnarbeiterklasse“ “(52) ersetzen will. Demgegenüber hebt er die Bedeutung des Mittelklassenbegriffs hervor und zieht den Schluß, ,daß die absolute und relative Ausdehnung der Mittelklassen das Klassengefüge der bürgerlichen Gesellschaft von Beginn an charakterisiert" (53). Die Lohnarbeit als produktive dürfe nicht mit ihrer verallgemeinerten Form gleichgesetzt werden. Die Marxsche These von der relativen Abnahme des Proletariats und der relativen Zunahme der Mittelklassen sei keineswegs irrig, sie folge als klassentheoretische Konsequenz aus den Gesetzen der kapitalistischen Akkumulation.

Auch Kostede betont die Uneinheitlichkeit dieser Mittelklassen und hebt hervor, daß die Verwendung dieses Begriffs keineswegs die Perspektiven gemeinsamer Aktion der Lohnarbeiter verschütte.

„Der Begriff der Mittelklassen muß also nicht zwangsläufig die spezifischen Verhältnisse produktiver, kommerzieller und unproduktiver Arbeiter verschütten, ebenso wie er es uns beläßt, die in der gemeinsamen Form der Lohnarbeit begründeten einheitlich erfahrbaren Widersprüche im gemeinsamen Kampf der Lohnarbeiter um die Erhaltung der menschlichen Arbeitskraft, der Sicherung, Verteidigung oder Erweiterung der jeweiligen Revenuen etc. aufzuspüren" (54).

Aus diesen Erkenntnissen folgt für Kostede, daß bei der Analyse der revolutionären Klassen nicht vom Begriff der Lohnarbeit schlechthin, sondern von der mehrwertproduzierenden produktiven Lohnarbeit auszugehen sei. Daraus ergebe sich folgender klassenanalytischer Gang, der auch das Verhältnis von Klassenanalyse und revolutionärer Taktik einschließe:

„Ausgehend von und auf der Basis der Marxschen Darstellung der Bewegungen und Strukturen der Klassen der bürgerlichen Gesellschaft im Nachvollzug der Bewegungen kapitalistischer Akkumulation will diese Konzeption in der Analyse realer Klassenkämpfe hinterfragen, welche Bereiche und Schichten der produktiven Klasse und der modernen Mittelklassen den zugespitzten und vielfältigen Torturen der kapitalistischen Verwertungszwänge unterliegend und in der Hetze und bornierten Arbeitsteiligkeit wirklicher Arbeitsprozesse geknechtet zum immer allein realen und historischen revolutionären Subjekt zusammengeschweißt werden. Erst die konkrete Geschichte der Klassenkämpfe formt das revolutionäre Subjekt zu einer wirklichen Einheit von ökonomischen, politischen und ideologischen Beziehungen und Zusammenhängen. Erst unter Einbezug dieser Ebene wird die Frage nach Strategie und Taktik beantwortbar, werden diese Antworten sich erweisen als revolutionär oder revisionistisch. Erst hier ordnet das Proletariat als wirkliches Subjekt seine eigenen Reihen und fragt andererseits nach Klassen und Schichten, die, obwohl nicht in antagonistischen Widersprüchen zum Kapital stehend, in mannigfachen mani-

A. a. O., S. 301

51 Akkumulation und Mittelklassen, in: Probleme des Klassenkampfs 13/1974

52 A. a. O., S. 1

53 A. a. O., S. 2

54 A. a. O., S. 10 
festen und sublimen Formen unter seiner erbarmungslosen Naturgesetzlichkeit und seinem blinden ,Fortschritt" leiden und im Voranschreiten der Klassenkämpfe auf die Seite des Proletariats gezogen werden können" (55).

Wichtiger noch als diese Beiträge zur Klärung des Mittelklassenbegriffs ist der methodische Ansatz Kostedes, die Herausbildung der Klassenstrukturen und ihre Charakterisierung ,als Reflex der inneren Widersprüche des Akkumulationsprozes$\operatorname{ses}^{\text {“ }}(56)$ zu begreifen und darzustellen, was er in den Hauptteilen seiner Arbeit versucht. Dieses Vorgehen, von der Akkumulation als ,,innerem Motor der Klassengliederung" auszugehen, rückt unserer Meinung nach zu Recht die Profitproduktion als begründendes und bewegendes Prinzip der Klassenstruktur in den Mittelpunkt der Klassenanalyse und stellt gegenüber bloß begrifflich-definitorischen marxistischen Arbeiten (57) und statischen Strukturanalysen (58) einen entscheidenden Fortschritt dar. Mit ihm ist auch eine weitere Annäherung an die geforderte und fällige Analyse der realen Klassenverhältnisse geleistet.

V.

Ich habe verschiedentlich hervorgehoben, daß ich allein im Ausgehen vom Produktions- und Reproduktionsprozesses des Werts die richtige klassenanalytische Vorgehensweise sehe. Aus ihr folgte fur die staatlichen Lohnarbeiter, daß sie das Kapital nicht reproduzieren, also nicht zur Arbeiterklasse gehören. Konstitutiv für ihre klassenmäßige Bestimmung ist, daß sie als Staatsagenten in Lohnform arbeiten (womit in der Regel die Abpressung von Mehrarbeit verbunden ist) und aus abgeleiteter Revenue bezahlt werden. Die Stellung beim Staat, d. h. neben und außerhalb des Kapitalverhältnisses, und die spezifische Quelle des Lohns umreißen die objektive Stellung der staatlichen Lohnarbeiter neben und zwischen Arbeiterklasse und Kapitalistenklasse. Sie sind deshalb (zusammen mit anderen unproduktiven, aus abgeleiteter Revenue bezalten Lohnarbeitern, z. B. in Gewerkschaften, Parteien, Kapitalverbänden) als lohnarbeitende Mittelklasse anzusprechen. Sie unterscheiden sich so auch klar von der kleinbürgerlichen Mittelklasse (Handwerker, Bauern, Kleinhändler, „, freie Berufe" usw.), die ihre Arbeit als Produktionsmittelbesitzer verrichtet.

Von dieser Klassenbestimmung der staatlichen Lohnarbeit ausgehend, gewinnt dann auch der Begriff der Lohnarbeiterklasse wieder eine begrenzte Bedeutung. Wenn auch die Lohnform nicht klassenkonstituierend ist, wie wir gesehen haben, so stellt sie doch den Hebel der spezifischen Bündnismöglichkeit zwischen Arbeiterklasse und lohnarbeitender Mittelklasse dar - und zwar als die Bedingungen des Arbeitsprozesses und der Abpressung von Mehrarbeit vermittelnde (und verhül-

55 A. a. O., S. $10 \mathrm{f}$. Das hier ausgedrückte Verhältnis von Theorie und Geschichte wird in ähnlicher Form öfters benannt, wenn es auch beim methodischen Postulat bleibt. Vgl. aber den Versuch der Konkretisierung in: links Nr. 60 und 61197

56 A. a. O., S. 2 . Vgl. auch S. 33, 40

57 Als solche muß trotz ihrer Qualität in der begrifflicher Schärfe auch die Arbeit des Projekts Klassenanalyse begriffen werden.

58 Das träfe auch auf Jung zu. 
lende) Form der Verausgabung von Arbeit. Diese inhaltliche Bestimmung des Lohns schließt die Tendenz zur Entleerung der Arbeit und zur Gleichgültigkeit des Arbeiters (59), zur Verstümmelung der Arbeiter im zergliederten Arbeitsprozeß, die Existenz des Arbeiters als Existenz nur für die Kapitalverwertung, ein. Wenn diese Bestimmungen auch im Produktions- und Reproduktionsprozeß des Kapitals ausgeprägt werden, so treten sie doch auch mit ähnlichen Charakteristika in der nichtproduktiven Sphäre des Staates auf und stellen das Scharnier eines Bündnisses dar, in dem die Arbeiterklasse die lohnarbeitende Mittelklasse bzw. Teile von ihr im konsequenten Kampf gegen die Ausbeutung stärkt und für den Sozialismus gewinnen kann (60).

Um Grad und Form der Bündnisfähigkeit der staatlichen Lohnarbeiter als Teil der lohnarbeitenden Mittelklasse genau zu bestimmen, muß u. a. allgemein und konkret-historisch untersucht werden, welcher Inhalt (gesellschaftliche Stellung, Funktion, Arbeitsinhalt, Bewußtsein) unter der Lohnform der Arbeit staatlicher Lohnarbeiter steckt. Dabei werden Möglichkeiten und Schranken des Bündnisses deutlicher werden. Dies freilich erst im Zusammenhang mit den realen Tendenzen des Klassenkampfs.

Der Staat zieht zur Erfüllung seiner Aufgaben Teile des gesellschaftlichen Waren- und Arbeitsfonds an sich. Die Größe dieser Teile hängt vom Umfang der Aufgabeund den ökonomischen Bedingungen (Akkumulationszyklus, Konkurrenz, Produktivität der Arbeit usw.) ihrer Erfüllung ab, konkret setzt sie sich erst über die Kämpfe der Klassen durch.

Die Analyse der Form und Funktionen des bürgerlichen Staates (61) liefert implizit Beiträge zur Bestimmung der staatlichen Lohnarbeit. Denn die Untersuchung der Rolle der unterschiedlichen Staatsfunktionen im Reproduktionsprozeß des Kapitals muß für die staatliche Lohnarbeit, die diesen Funktionen jeweils zugeordnet ist, Konsequenzen haben. Der innere Widerspruch des Staates in der bürgerlichen Gesellschaft, formal die Gleichheit aller Individuen anzuerkennen, tatsächlich aber Ungleichheit und Klassenherrschaft zu garantieren, muß auch die staatliche Lohnarbeit prägen. Ihre Tätigkeit trägt nicht nur einfach zur Reproduktion der Gesellschaft bei, sie trägt zur Reproduktion der kapitalistischen Klassengesellschaft bei. Sie vermittelt nicht nur Qualifikation und Gesundheit des Arbeitsvermögens, sie vermittelt auch direkt die Ideologie und die Repression der herrschenden Klasse.

59 Zur Kategorie der Gleîchgültigkeit vgl. Autorenkollektiv am Institut für Soziologie der FU Berlin, Klassenlage und Bewufstseinsformen technisch-wissenschaftlicher Lohnarbeiter, Frankfurt a. M. 1973, S. $115 \mathrm{ff}$. und $134 \mathrm{ff}$.

60 Ähnlich führt Kostede aus, ,daß die notwendige Verallgemeinerung der Form der Lohnarbeit über den kapitalistischen Produktionsprozeß hinaus für die Analyse der Widersprüche der realen Klassenkämpfe von eminenter Bedeutung ist und đieser Aspekt im Begriff der Lohnarbeit uns einen kategorialen Schlüssel für das Aufdecken und die Darstellung der realen Zwänge und Herrschaftsformen gibt, denen zunehmend auch diese Bevölkerungsteile der bürgerlichen Gesellschaft im zyklischen Gang der Akkumulation und in der arbeitsteiligen Verödung und Entleerung der wirklichen kommerziellen und unproduktiven Arbeitsprozesse ausgeliefert sind". A. a. O., S. 8.

$61 \mathrm{Vgl}$. Blanke, Jürgens, Kastendiek: Zur neueren marxistischen Diskussion um Form und Funktion des bürgerlichen Staates, Probleme des Klassenkampfs, 14-15/1974 
Das hängt $u$. a. von ihrer Stellung innerhalb der staatlichen Funktionen ab, also von ihrer konkreten Tätigkeit und ihrer Stellung in der Hierarchie. Die staatlichen Lohnarbeiter realisieren mit dem Inhalt ihrer Tätigkeit die Funktionen des Staates.

Staatliche Lohnarbeit ist unproduktive Arbeit. Sie tauscht sich gegen staatliche Revenue, die im wesentlichen aus Steuern zusammengesetzt ist, aus, gegen Geld als Geld und nicht gegen Geld als Kapital. Der Käufer kann zwar ihren Gebrauchswert konsumieren, aber die Konsumtion dieses Gebrauchswert liefert ihm keinen Mehrwert. Dies widerum liegt an der ökonomischen Formbestimmung der Staatstätigkeit als im wesentlichen nicht-kapitalistisch, d.h.: der Staat verwendet eine bestimmte Geldmasse zum Ankauf von Produktionsmitteln und Lohnarbeitern, nicht um Kapital, die um den Mehrwert bereicherte Ausgangssumme, zu produzieren. Er verwendet diese Geldmasse, um Produktionsmittel und Lohnarbeiter zu kaufen, die die für die kapialistische Gesellschaft notwendigen Dienste möglichst ökonomisch produzieren.

Die mit der Entwicklung des Kapitalverhältnisses sich verallgemeinernde Durchsetzung der Lohnarbeit hat auch Verhältnisse erfaßt, die ihrem strengen Begriff als dem Kapital gegenübergesetzte Lohnarbeit widersprechen. Lohnarbeit existiert nicht nur in der Produktion und in der Zirkulation, sondern auch beim Staat. D. h. die beim Staat verrichtete Arbeit hat die Form der Lohnarbeit angenommen und ist den die Lohnhöhe regelnden Gesetzen unterworfen, wenn auch modifiziert. Der Lohn der staatlichen Lohnarbeiter ist wohl in bestimmten Perioden unterdurchschnittlich, das wird aber ausgeglichen durch die Sicherheit des Arbeitsplatzes und langfristig bzw. indirekt zu Buche schlagende Lohnteile (u. a. günstige Altersversorgung, verbilligter Bezug öffentlicher Dienste). Andererseits ist diese staatliche Lohnarbeit eben nicht wie in der Produktion und Zirkulation kapitalproduzierend und auch nur vermittelt dem Auf und $\mathrm{Ab}$ des kapitalistischen Akkumulationszyklus ausgesetzt, was allerdings für die verschiedenen Bereiche staatlicher Lohnarbeit sehr unterschiedlich aussieht. Für die staatlichen Lohnarbeiter heißt das: es wird zwar Arbeitskraft gegen Lohn verkauft wie in der Industrie auch, aber beim Gebrauch dieser Arbeitskraft handelt es sich nicht um die direkte Errfahrung der Profitproduktion. Arbeitsbelastung und Arbeitsanspannung sind nicht so leicht auf einen „Output“ zu beziehen, da - mit Ausnahme der Staatsbetriebe, auf die ohnehin viele der hier angeführten Bestimmungen nicht oder nur teilweise zutreffen keine zirkulationsfähigen Waren produziert werden (62). Die Effektivität der staatlichen Dienste mißt sich nicht am Markt. Die Anforderungen an Staatsdienste ergeben sich zum guten Teil außerhalb der Staatssphäre. Der Staat kann hier zwar modifizieren, im wesentlichen aber kann er nur reagieren, und eine seiner Reaktion ist die Ökonomisierung und Rationalisierung der staatlichen Lohnarbeit - um dem Anforderungsdruck nachzukommen bzw. die faux frais zu senken, nicht um Kapital zu produzieren. Der konkret-nützliche Gebrauchswertcharakter der Arbeit steht objektiv und subjektiv im Vordergrund.

Weitbrhin ist dem staatlichen Lohnarbeiter die Quelle seiner Bezahlung, der industriell produzierte Neuwert (über Steuern), verschleiert. Ihm erscheint der Staat

Von der Effizienz bürgerlicher Verwaltungswissenschaft einmal abgesehen. 
als „Arbeitgeber“, der Staat als der Gesellschaft neben- oder übergeordnete Institution, nicht die sozialen Klassen. Der staatliche Lohnarbeiter teilt daher auch die Illusion des Staates über sich selbst, Hüter und Diener des Gemeinwohls zu sein. Noch anders ausgedrückt: im Bewußtsein des staatlichen Lohnarbeiters erscheint der Staat nicht als Ausdruck der bürgerlichen Gesellschaft und eingebannt in ihre Grenzen, sondern als aktive und ordnungsstiftende Kraft gegenüber der Gesellschaft.

Für diejenigen, aus deren Revenue der Staat unterhalten wird (Kapital und Lohnarbeiter des Kapitals), stellen sich eben die Tätigkeiten staatlicher Lohnarbeiter nicht nur als konkret-nützliche dar, sondern auch als Abzüge von ihrem Profit bzw. Lohn. Insofern erscheint ihnen alle Arbeit beim Staat als unterschiedslose. Aus dem Interesse an möglichst geringen Steuern resultiert der Druck vor allem der Kapitaleigner auf den Staat, milder oder schärfer je nach dem Gang der Akkumulation, die Kosten seiner Funktionen zu ökonomisieren. Auf die staatlichen Lohnarbeiter schlägt sich das in der Form nieder, daß sie vom Staat gezwungen werden, die über ihre Reproduktion hinausgehende Mehrarbeit zu erhöhen, um damit die Personalkosten zu drücken.

Ist auch die Arbeit der staatlichen Lohnarbeiter von der Gebrauchswertseite her bestimmt, so tritt doch der gesamtkapitalistische Verwertungsprozeß nicht ganz zurück. In der Quelle des Lohns der Arbeit beim Staat ist die Möglichkeit des steigenden vermittelten Drucks der gesellschaftlichen Grundklassen eingeschlossen. Das kann nicht nur zu Brüchen im Bewußtsein der staatlichen Lohnarbeiter führen, die sich mit dem Inhalt ihrer Tätigkeit identifizieren, durch zunehmende Verknappung der finanziellen Mittel aber die Ziele ihrer Arbeit nicht erreichen können (z. B. soziale Rehabilitation) und selbst materiell schlechter gestellt werden. Das kann zum Widerspruch zwischen Inhalt und Form der Arbeit auch dergestalt führen, daß etwa dem Polizisten der "Schutz der öffentlichen Ordnung“ nicht mehr ausreichend vergütet erscheint, was ein Ansatz der Erschütterung seiner Staatsloyalität ist. Für die Masse der staatlichen Lohnarbeiter in den verschiedenen Bereichen staatlicher Tätigkeit wird sich eine Verengung des finanziellen Spielraumes des Staates in Lohndruck, geringerer Arbeitsplatzsicherheit, Steigerung der Arbeitshetze, Abbau ihrer noch verbliebenen Privilegien und zunehmender politischer Disziplinierung äußern. Es tritt also eine Annäherung ihrer Lebenslage an die der übrigen Lohnarbeiter ein. Die Verwendung des Begriffs ,staatliche Lohnarbeit" - trotz aller gemachten Einschränkungen - bedeutet insofern ein wissenschaftliches und politisches Programm, als hinter der erscheinenden Bewegung die Relevanz der Lohnform für die reale Situation der beim Staat Beschäftigten aufzuspüren ist und über sie die Verbindung der staatlichen Lohnarbeiter zu der Arbeiterklasse geklärt werden soll.

Es darf auch nicht übersehen werden, daß der oben skizzierten Möglichkeit der Annäherung der staatlichen Lohnarbeiter an die übrigen Lohnarbeiter einige Tendenzen entgegenstehen. $\mathrm{Da}$ ist zunächst die schon erwähnte Bindung an die Inhalte der Arbeit, die zusammen mit deren objektiver Funktion teilweise den Interessen der Arbeiterklasse entgegenstehen muß, solange die bürgerliche Gesellschaft existiert. Weiterhin wird der Staat versuchen, durch Bestechungen und öko- 
nomisch-politischen Druck sein Personal bei der Stange zu halten. Bei einer Reihe von staatlichen Lohnarbeitern, vor allem im höheren Verwaltungsapparat, versteckt sich unter der Lohnform nur das leitende politische Personal der Bourgeoisie. Sie sind auch nicht mehr durch (vermittelte) Gesetze der Lohnarbeit, sondern höchstens durch die der politischen Konjunktur bestimmt und erhalten beträchtliche politische Zusatzgratifikationen. Auf die allgemeinen realen und bewußtseinsmäßigen Unterschiede der staatlichen Lohnarbeiter im Verhältnis zu den übrigen Lohnarbeitern ist oben schon eingegangen worden. Hierher gehören auch die objektiven Interessengegensätze von Arbeiterklasse und staatlichen Lohnarbeitern aufgrund des Steuerwesens. Insgesamt handelt es sich hier um Unterschiede und Gegensätze, welche über den - durch die Konkurrenz unter den Arbeitern und die Verschiedenheit der kapitalistischen Produktionsprozesse erzeugten - Charakter einer Fraktionierung innerhalb einer Klasse weit hinausgehen.

Die verschiedenen Staatsfunktionen haben eine unterschiedliche Bedeutung für den gesellschaftlichen Reproduktionsprozeß, eine Differenzierung, die sich in der Bewegung der historischen Kapitalakkumulation und der aus ihr hervorgetriebenen sozialen Konflikte noch verschärft. Die konkret-historische Analyse wird klären, welche Staatsfunktionen wann, in welchem Ausmaß und mit welchen finanziellen Modalitäten und Schranken wahrgenommen werden. In ihr wird auch zu zeigen sein, daß die verschiedenen Abteilungen der staatlichen Lohnarbeiter in unterschiedlicher Weise von der ökonomischen und politischen Bewegung der bürgerlichen Gesellschaft betroffen sind. Hier wäre sowohl die Bedeutung tradierter Ideologie und Beschäftigungsformen in den nationalen Gesellschaften (z. B. Beamtentum) zu berücksichtigen, wie auch stärker nach Arbeitsbereichen und Stellung in der Hierarchie zu differenzieren ist. 